\section{REFERENCES}

1 Solovic I, Sester M, Gomez-Reino JJ, et al. The risk of tuberculosis related to tumour necrosis factor antagonist therapies: a TBNET consensus statement. Eur Respir J 2010; 36: 1185-1206.

2 Mack U, Migliori GB, Sester M, et al. LTBI: latent tuberculosis infection or lasting immune responses to $M$. tuberculosis? A TBNET consensus statement. Eur Respir J 2009; 33: 956-973.

3 Diel R, Goletti D, Ferrara G, et al. Interferon- $\gamma$ release assays for the diagnosis of latent Mycobacterium tuberculosis infection: a systematic review and meta-analysis. Eur Respir J 2011; 37: 88-99.

4 Lange C, Rieder HL. Intention to test is intention to treat. Am J Respir Crit Care Med 2011; 183: 3-4.
5 Leung CC, Rieder HL, Lange C, et al. Treatment of latent infection with Mycobacterium tuberculosis: update 2010. Eur Respir J 2011; 37: 690-711.

6 Erkens CG, Kamphorst M, Abubakar I, et al. Tuberculosis contact investigation in low prevalence countries: a European consensus. Eur Respir J 2010; 36: 925-949.

7 Sester M, van Leth F, Girardi E, et al. Head-to-head analysis of IGRAs and skin-testing in immunocompromised patients: interim analysis of a multicenter TBNET study. Eur Respir J 2010; 36: Suppl. 54, 370s-371s.

8 Tsiouri G, Gaitanis G, Kiorpelidou D, et al. Tuberculin skin test overestimates tuberculosis hypersensitivity in adult patients with psoriasis. Dermatology 2009; 219: 119-125.

DOI: 10.1183/09031936.00041811

\title{
Internal consistency of reference equations
}

\section{To the Editors:}

In a recent European Respiratory Journal paper addressing the choice of reference values for spirometric indices, mean $\mathrm{z}$ scores were used to quantify deviation from a large collated data set or to establish the absence of secular trends in forced expiratory volume in $1 \mathrm{~s}$ (FEV1) or forced vital capacity (FVC) [1]. Yet, once an appropriate set of reference equations is selected for use in any given laboratory, it is equally important to assess whether these equations are internally consistent, e.g. in terms of age dependency of the various parameters under study. When consulting the most recent standardisation documents for guidance on the choice of reference equations $[2,3]$ the American Thoracic Society (ATS)/European Respiratory Society (ERS) Task Force literally states "currently this committee does not recommend any specific set of equations for use in Europe". Due to our laboratory's particular geographical location, we have thus far felt compelled to apply the European Community for Steel and Coal (ECSC) equations for adult spirometry and lung volumes. Through the various updates up to the 2005 ATS/ERS recommendation [2], many reference equations date back to the original 1983 document [4], which was compiled from all the available adult data at the time. Applying these equations, we are now faced with two cases of internal inconsistency.

While the age- and height-dependent prediction equations for FEV1 and FVC separately lead to a predicted FEV1/FVC value which is similar to the one computed directly from the FEV1/ FVC prediction equation in male subjects, this does not hold true for females. For a $175 \mathrm{~cm}$ tall male ranging $25-65 \mathrm{yrs}$, the difference between the predicted FEV1/FVC value and the ratio of predicted FEV1 and predicted FVC values ranges 2.0$2.5 \%$. In a $165 \mathrm{~cm}$ female aged $65 \mathrm{yrs}$, the difference amounts to as much as $7.2 \%$; indeed, the ratio of predicted FEV1 and predicted FVC is $84 \%$, as opposed to the predicted FEV1/FVC value of $76.8 \%$. Alternatively, the Third National Health and Nutrition Examination Survey (NHANES III) reference equations for Caucasians [5] lead to corresponding differences in FEV1/FVC of $\leqslant 0.1 \%$, for both sexes across the same age range. In comparison with the NHANES III prediction equations, both FEV1 and FVC prediction equations are quite different, yet, the prediction equation for $\mathrm{FEV} 1 / \mathrm{FVC}$ is very similar to the ECSC one. This indicates that it is the relative age dependence of FEV1 and FVC in females which is in error, due to an inaccuracy in either age dependence of FEV1, in age dependence of FVC or in both. This inconsistency in the reference equations, or its derived limits of normal, is relevant for instance when determining prevalence of restriction (FVC) and obstruction (FEV1/FVC) in the same population. Alternatively, when assessing both the presence of obstruction (FEV1/FVC) and its severity (FEV1), or quantifying lung age, based on either predicted FEV1 or predicted FEV1/FVC, an inconsistency between FEV1/FVC and its components is likely to bias the outcome.

Another apparent discrepancy shows up in the ECSC reference equations of functional residual capacity (FRC), showing a very poor dependency on age in adult females and a nine-fold greater age-dependence in adult males. This is in contrast to a similar age dependence for boys and girls through to adulthood [3], and also in contrast to the reference equation of the largest of the data set on which the composite FRC reference equation is based, namely the 1,841 subjects investigated by W.J. Ulmer and co-workers as referenced by QUANJER [4]. These authors showed a similar age dependence for both sexes: a coefficient that is the same as in the ECSC equations for FRC in males, and an age coefficient in females being $78 \%$ of the coefficient in males. Obviously, the virtual absence of age dependence in predicted FRC for females in the ECSC reference equation may unduly identify an older female subject as hyperinflated. This may be particularly relevant to diseases and treatments involving hyperinflation, which make use of FRC or inspired capacity as an indicator.

We suspect that both these observations are merely the result of understandable limitations of $\sim 30$-yr-old reference equations, that are composite equations based on even older reference equations, and not on collated data sets of individual data points as suggested now [1]. This should encourage any current or future initiative to pool as many reliable data as possible before extracting reference equations, thereby avoiding 
a posteriori internal inconsistencies. The aforementioned problems associated with the ECSC reference equations in particular should be a particular stimulus for gathering updated data on spirometry and on lung volume across the (expanded) European community.

\section{S. Verbanck*, J. Vandevoorde ${ }^{\#}$ and W. Vincken* \\ ${ }^{*}$ Respiratory Division, and "Dept of General Practice, Aca- demic Hospital, University of Brussels, Brussels, Belgium.}

Correspondence: S. Verbanck, UZ Brussel, Laarbeeklaan 101, Brussels, Belgium. E-mail: sylvia.verbanck@uzbrussel.be

Statement of Interest: None declared.

\section{REFERENCES}

1 Quanjer PH, Stocks J, Cole TJ, et al. Influence of secular trends and sample size on reference equations for lung function tests. Eur Respir $J$ 2011; 37: 658-664.

2 Pellegrino R, Viegi G, Brusasco V, et al. Interpretative strategies for lung function tests. Series ATS/ERS Task Force: Standardisation of lung function testing. Eur Respir J 2005; 26: 948-968.

3 Stocks J, Quanjer PH. Reference values for residual volume, functional residual capacity and total lung capacity. ATS Workshop on Lung Volume Measurements. Official Statement of The European Respiratory Society. Eur Respir J 1995; 8: 492-506.

4 Quanjer PH. Standardized lung function testing. Bull Eur Physiopathol Respir 1983; 19: 5-95.

5 Hankinson JL, Odencrantz JR, Fedan KB. Spirometric reference values from a sample of the general US population. Am J Respir Crit Care Med 1995; 152: 179-187.

DOI: 10.1183/09031936.00022411

\section{From the authors:}

The summary equations published by the European Community for Steel and Coal (ECSC) and the European Respiratory Society (ERS) [1, 2] are an average of prediction equations published before 1983; arising from this they have serious deficiencies. All ECSC studies were paid for by levies on coal and steel, and thus lacked adequate studies in females. Since no appropriate data existed for females, and no funds were available for a new study, the working party used published prediction equations derived from healthy nonsmokers to generate a new database, and then used this to derive a new equation. The limitations of this technique are quite obvious, some of these were discussed in the 1983 report [1]. Table XVIII of that report also summarises the inconsistencies that arise from deriving the forced expiratory volume in $1 \mathrm{~s}$ (FEV1)/forced vital capacity (FVC) ratio from predicted FEV1 and predicted FVC, compared with a predicted ratio, the latter being a more robust and appropriate approach.

As to the noted discrepancies with respect to functional residual capacity (FRC)/total lung capacity (TLC), the 1983 report noted: "For the FRC and FRC/TLC, the situation is unsatisfactory [...] there is wide variability in predicted FRC according to various authors, and therefore this index is not likely to be suitable for diagnostic purposes. The predicted FRC/TLC ratio is based on only three discrepant sources; at the present time, the summary equation for this ratio cannot be recommended for routine use." Despite these explicit warnings within the document, the ECSC equations and other outdated and inappropriate equations are readily available in lung function software and thus continue to be used in a blinkered fashion in both clinical and research settings.

Deriving new prediction equations should ideally be based on recent and representative data. New studies are, however, very costly because large numbers are required to obtain good population estimates [3]. Collation of available data, obtained with state-of-the-art techniques, is a valid and cheap alternative $[4,5]$. Unfortunately, although equations for spirometry which span the 3-80 yrs age range in white subjects have been available for several years [5]; their availability in clinical practice has been limited because they have not been implemented widely by manufacturers. Use of the new equations [5], derived from good quality studies with state-of-the-art analytical techniques, should now be standard practice, and several key manufacturers have recently upgraded their software to facilitate this approach. Desktop software (Microsoft Windows) based on the work of STANOJEVIC et al. [5] is available for free from www.lungfunction.org/growinglungs/software.html.

Over the past decade, research groups and funding agencies have raised the importance of data sharing for scientific purposes. Arising from this, the Global Lungs Initiative (www. lungfunction.org), an ERS task force endorsed by the American Thoracic Society, the Australian and New Zealand Society of Respiratory Science, the Asian Pacific Society of Respirology and the American Association of Chest Physicians, will this year issue new reference values for spirometry based on data from nearly 100,000 healthy subjects (age range 3-95 yrs) from 70 centres worldwide, encompassing a range of ethnic groups. Hopefully these will be adopted internationally, to overcome the problems that S. Verbanck and co-workers describe. Future initiatives from this task force will hopefully address remaining challenges with respect to lung volumes and diffusion capacity of the lung for carbon monoxide.

\section{P.H. Quanjer, J. Stocks and S. Stanojevic}

Portex Respiratory Unit, UCL Institute of Child Health, London, UK.

Correspondence: J. Stocks, Portex Respiratory Unit, UCL Institute of Child Health, 30 Guilford St, London, WC1N 1EH, UK. E-mail: j.stocks@ich.ucl.ac.uk

Statement of Interest: None declared.

\section{REFERENCES}

1 Quanjer PH Standardized lung function testing. Report Working Party Standardization of Lung Function Tests, European Community for Steel and Coal. Bull Eur Physiopathol Respir 1983; 19: 45-51.

2 Quanjer PH, Tammeling GJ, Cotes JE, et al. Lung volumes and forced ventilatory flows. Report Working Party Standardization of Lung Function Tests, European Community for Steel and Coal. Official Statement of the European Respiratory Society. Eur Respir J 1993; 6: S5-S40. 